\title{
Mathematical and Synoptic Aspects of a Small-Scale Wave Disturbance Over the Lower Great Lakes Area
}

\author{
Howard L. FERGUSON \\ Air Services Meteorological Branch, Department of Transport, Toronto, Canada
}

(Manuscript received 3 May 1966, in revised form 28 February 1967)

ABSTRACT

\begin{abstract}
A squall line which crossed the lower Great Lakes area on 13 March 1963 is described. Characteristics of the squall line are compared to typical features of such phenomena deduced in other studies. The instability line was unusual in terms of the associated surface weather. It extended for a considerable distance over a low-level Arctic frontal zone and had a propagation speed and length-of-life in excess of typical values. The disturbance was found to conform closely to the equations developed by Goldie for internal gravity waves of a somewhat smaller scale. Favorable conditions for the development of such wave phenomena are discussed briefly, together with their possible implications in synoptic-scale analysis and forecasting.
\end{abstract}

\section{Introduction}

Between 1200 and 1300 GMT on 13 March 1963 a short-period pressure oscillation was recorded on the barograph at Centralia, Ontario (CE). The main fluctuation consisted of a pressure fall of about $4 \mathrm{mb}$ in a 10 -min period followed by a pressure rise of about $3 \mathrm{mb}$ in $30 \mathrm{~min}$ (Fig. 1). The surface weather observations (Table 1) showed a marked variation in wind speed and direction between 1130 and 1300 GMT. Ice pellets were reported briefly, preceded by a combination of fog and snow. No thunder was reported. The synoptic-scale analyses available gave no indication of why such a pressure fluctuation occurred. The observed weather conditions differed markedly from those usually associated with instability lines.

A study was undertaken to determine the characteristics of the phenomenon of interest in operational analysis and forecasting (Ferguson, 1964). Further investigation revealed that the behavior of the wave disturbance conformed closely to the behavior of smaller-scale internal gravity waves studied by Goldie (1925). Mathematical characteristics of the wave disturbance were determined using Goldie's equations.

\section{Observed characteristics of the instability line}

The surface chart for 1200 GMT 13 March (Fig. 2) showed a complex low pressure area over Indiana and Ohio. A quasi-stationary Arctic front extended from northern New York to a wave in northern Indiana, with a diffuse cold front southwestward through Arkansas. The radiosonde reported for Flint (FNT), Dayton (DAY), and Buffalo (BUF), are superimposed in Fig. 3. The points $F$ and $F^{\prime}$ in the figure represent the positions of the frontal surface over Flint and

TABLE 1. Summary of weather observations at Centralia, Ontario (CE), 13 March 1963. Low stratus and stratocumulus ceilings were observed throughout the period.

\begin{tabular}{|c|c|c|c|c|c|}
\hline$\underset{(\mathrm{GMT})}{\mathrm{Time}}$ & Weather & $\underset{(\mathrm{mb})}{\text { MSL pressure }}$ & $\begin{array}{l}\text { Temperature } \\
\left({ }^{\circ} \mathrm{F}\right)\end{array}$ & Direction & $\begin{array}{l}\text { Wind } \\
\quad \text { Speed (kt) }\end{array}$ \\
\hline 1100 & fog & 1012.6 & 33 & 020 & 3 \\
\hline 1132 & snow, fog & & & 340 & 5 \\
\hline 1142 & ice pellets, fog & & & 360 & 3 \\
\hline 1200 & & 1011.9 & 33 & 070 & 12 \\
\hline 1206 & & & & 070 & 18 gusts to 29 \\
\hline 1209 & & & & 070 & 24 gusts to 35 \\
\hline 1213 & & & & 070 & 26 gusts to 37 \\
\hline 1223 & $\begin{array}{l}\text { no fog or } \\
\text { precipitation }\end{array}$ & $\begin{array}{l}\text { (pressure } \\
\text { falling } \\
\text { ranidly) }\end{array}$ & & 070 & 24 gusts to 36 \\
\hline 1300 & & $\begin{array}{l}1011.6 \\
\text { (pressure } \\
\text { unsteady) }\end{array}$ & 35 & 040 & 6 \\
\hline 1326 & snow flurries & & & 040 & 4 \\
\hline 1345 & & & & 040 & 8 \\
\hline 1400 & snow flurry, fog & 1012.4 & 33 & 360 & .6 \\
\hline
\end{tabular}




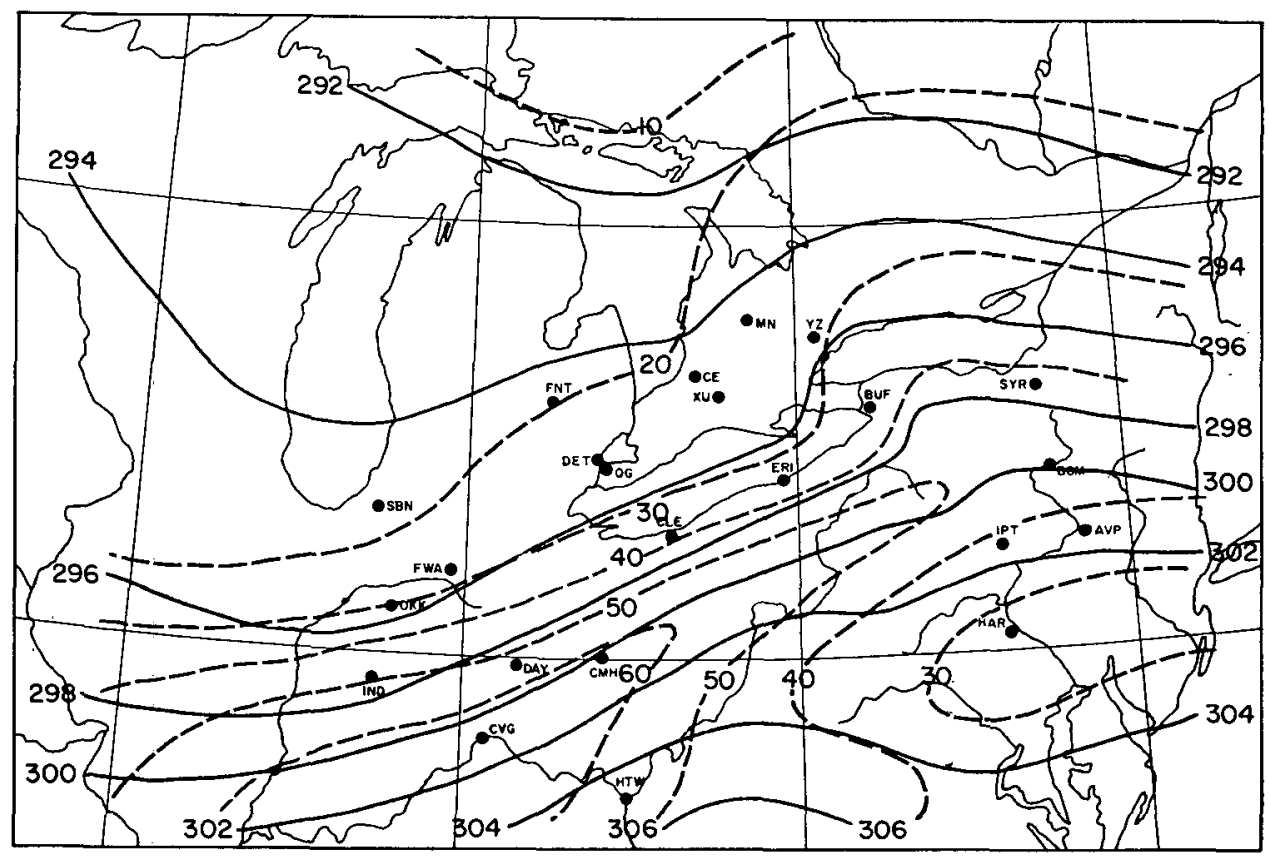

FIG. 9. 700-mb chart, 1200 GMT 13 March 1963. Solid lines are contours at 20-m intervals, dashed lines are isotachs at $10-\mathrm{kt}$ intervals.

$\beta=$ the angle between the direction of propagation of the wave disturbance and the mean wind direction in the lower layer,

$V=$ the propagation speed of the disturbance, and $\Delta U_{s}=$ the "orbital wind speed", i.e., the amplitude of the variation in surface wind speed during the passage of the wave. This is equivalent to the "outflow wind" discussed above.

Combining (2) and (3) we have

$$
\frac{\Delta P_{s}}{\rho \Delta U_{s}}=(U \cos \beta-V) .
$$

For the case under consideration, $\mathbf{U}$, the average wind velocity in the lower layer, was taken to be the gradient wind determined from the surface isobars (Fig. 2). This was found to represent an excellent vector average of the reported surface winds during the passage of the wave (Table 2 and Fig. 7). The velocity $\mathbf{V}$ of the wave over southwestern Ontario was found from the isochrone chart (Fig. 6). The value of $\beta$ was determined from the vector diagram (Fig. 7). The mean density $\rho$ of the lower layer was calculated assuming conditions similar to the radiosonde report at Flint (Fig. 3) and taking for an average pressure $900 \mathrm{mb}$ and for an average temperature $-3 \mathrm{C}$. The orbital wind speed $\Delta U_{s}$ was found from Fig. 7 . The following values were determined:

$$
\begin{aligned}
& \mathrm{U}=060^{\circ} / 18 \mathrm{kt} \\
& \mathrm{V}=260^{\circ} / 44 \mathrm{kt} \\
& \beta=160^{\circ}
\end{aligned}
$$

$(U \cos \beta-V)=61 \mathrm{kt}$

$$
\begin{aligned}
\rho & =1.16 \times 10^{-3} \mathrm{gm} \mathrm{cm}^{-3} \\
\Delta U_{s} & = \pm 19 \mathrm{kt}
\end{aligned}
$$

On substituting in (4) we find

$$
\Delta P_{s}=\mp 3.6 \mathrm{mb} \text {. }
$$

The signs indicate the relationship between the surface pressure and wind variations. Falling pressure occurred in conjunction with a "positive" orbital wind speed, i.e., with an orbital wind vector ( $\mathbf{Y}$ in Fig. 7 ) in the direction of $\mathbf{V}$. Rising pressure occurred with the onset of "negative" orbital wind speeds, i.e., with the strong east-northeasterly winds, as can be seen from an examination of Table 2 and Figs. 5 and 7.

The computed value of $3.6 \mathrm{mb}$ for the surface pressure variation is in good agreement with the observed pressure amplitude of about $3.3 \mathrm{mb}$ at Windsor (QG) indicated in Fig. 5. This represented a near-maximum observed value. The wave, behaving as a stable wave in terms of the surface pressure variation, gradually decreased in amplitude as it moved east-northeastward from the Windsor area. The observed pressure variation at Centralia was more pulse-like in character but had an estimated "amplitude" of about two-thirds the computed value.

The amplitude of the wave motion at the discontinuity (frontal) surface was determined using (3). Taking the depth $h$ of the lower stratum as $7000 \mathrm{ft}$ or $2140 \mathrm{~m}$ as indicated by the ascent curve for Flint (Fig. 3), we find from (3) an amplitude $A$ of $2150 \mathrm{ft}$ or $655 \mathrm{~m}$. This value represents the approximate amplitude of the oscillation of the frontal surface in the Flint-Windsor-Centralia region as a result of the motion of the wave.

The wavelength $\lambda$ of the disturbance was determined 
from

$$
\lambda=T V,
$$

where $T$ is the period of the disturbance. The barograph trace at Windsor (Fig. 5) suggests an initial period of about $45 \mathrm{~min}$ while at Toronto the period had increased to about $2 \mathrm{hr} 15 \mathrm{~min}$. Due to the ill-defined nature of the pressure wave away from the main impulse, these values should be regarded as rough approximations. From (5) we find a wavelength increasing with time from about $30 \mathrm{n} \mathrm{mi}$ in the Windsor area to about $100 \mathrm{n}$ mi near Toronto. Thus, while the propagation speed of the main pressure wave remained nearly constant with time, the amplitude decreased and the wavelength increased.

\section{Comparison of wave characteristics with other studies}

Cases similar to the one considered here have been studied by Goldie (1925), McRae and McGann (1965) and Gossard and Munk (1954). Goldie discussed cases similar to that investigated by Pothecary (1954), i.e., wave disturbances travelling east-northeastward over quasi-stationary frontal surfaces, the direction of propagation being parallel to the orientation of the front and opposite to the direction of the low-level flow. In these cases, the perturbations evidently occurred in association with strong shear, particularly directional shear, through the frontal layer. Goldie noted that "many cases occurred in easterly winds in front of depressions, i.e., under the 'Steering Surface' or 'warm front,' and a few even in the cold sector to the rear."

McRae and McGann found (2) and (3) applicable to wave disturbances occurring above sea breeze and land breeze boundaries and frontal zones in northwestern Australia. Gravity waves associated with sea breezes were found to propagate west-southwestward normal to the orientation of the sea breeze boundary. Fifty separate cases of such small-scale wave phenomena were observed by McRae and McGann in a 2-yr period.

In all of these studies, typical amplitudes of the surface pressure and wind oscillations were of the order of $0.5-1.0 \mathrm{mb}$ and $5-10 \mathrm{mph}$, respectively, and typical wavelengths computed were of the order of $5-15 \mathrm{~km}$. The phenomenon discussed here is evidently of a somewhat larger scale than the typical gravity-wave type of disturbance discussed by other authors. The time and space continuity suggest this, as does the fact that certain associated features of the disturbance are detectable by an observing network density which could be considered "small synoptic scale" rather than mesoscale.

The author has observed a number of cases of similar small-scale pressure oscillations of $1 \mathrm{mb}$ or more. It appears that over the Great Lakes area favorable conditions are found in the spring of the year and in synoptic situations typified by the example discussed in this paper and illustrated in Fig. 2. However, similar pressure fluctuations have been observed with anticyclonic flow and clear skies.

Disturbances of the type studied here are capable of producing longer wavelength perturbations in objectively analyzed fields of contour height, divergence and vorticity through "aliasing" (Blackman and Tukey, 1958). As indicated by Eddy (1963), the effect on the contour field is usually small in terms of wave energy. The effect on divergence and vorticity patterns may be appreciable, however, particularly when the small-scale disturbance takes the form of a long symmetric squall line. There is, in addition, the possibility that such disturbances may occasionally grow into significant synoptic-scale baroclinic waves.

\section{Conclusions}

The small-scale wave disturbance which crossed the lower Great Lakes on 13 March 1963 had characteristics intermediate between typical mesoscale and synopticscale phenomena. Though identifiable as an instability line and decidedly larger than typical gravity waves, this disturbance was found to conform closely to equations developed by Goldie (1925) for mesoscale waves occurring on a boundary between a shallow, stable lower layer and a superimposed, infinitely deep layer of differing mean flow in an incompressible fluid. The squall line produced significant weather variations of interest in the problem of short-range forecasting.

A synoptic situation similar to that shown in Fig. 2, appears to be favorable for the occurrence of small-scale wave disturbances, especially in late winter. It is possible that these disturbances may occasionally be of significance in the development of new synopticscale "short-wave" troughs and ridges and associated phenomena. They are at least of a magnitude sufficient to produce small-scale variations or "noise" in computed synoptic-scale absolute vorticity patterns.

\section{REFERENCES}

Blackman, R. B., and J. W. Tukey, 1958: The Measurement of Power Spectra. New York, Dover Publications Inc., 190 pp.

Boucher, R. J., and R. Wexler, 1961: The motion and predictability of precipitation lines. J. Meteor., 18, 160-171.

Eddy, A., 1963: Kinematic divergence and large-scale energy conversion. Sci. Rept., McGill University, 8, 45-47.

Ferguson, H. L., 1964: An unusual instability line over southwestern Ontario, March 13, 1963. Meteorological Branch, Department of Transport, Canada, CIR-3982, $9 \mathrm{pp}$.

Goldie, A. H. R., 1925: Waves at an approximately horizontal surface in the atmosphere. Quart. J. Roy. Meleor. Soc., 51, 239-246.

Gossard, E., and W. Munk, 1954: On gravity waves in the atmosphere. $J$. Meteor., 11, 259-269.

McRae, J. N., and J. J. McGann, 1965: Periodic fluctuations in wind, pressure and temperature at Port Hedland. A ustralian Meleor. Mag., No. 48, 1-7.

Pothecary, J. J. W., 1954: Short-period variations in surface pressure and wind. Quart. J. Roy. Meteor. Soc., 80, 39j-401.

Tepper, M., 1959: Mesometeorology-the link between macroscale synoptic weather and local weather. Bull. Amer. Meteor. Soc., 40, 56-72.

Wilson, J. W., and E. Kessler III, 1963: Use of radar summary maps for weather analysis and forecasting. $J$. Appl. Meteor., 2, 1-11. 